Noma 2016, 34(2), 97-106

Revista de Psicologia, Ciències de l’Educació i de l'Esport

ISSN: 1138-3194

Copyright (c) 2016

www.revistaaloma.net

\title{
La implicación de la familia en la formación ético-cívica de los jóvenes a través de las TIC
}

\author{
Jesús Plaza \& Carmen Caro \\ Universidad Internacional de La Rioja
}

Recibido: 25-2-2016

Aceptado:1-9-2016

La implicación de la familia en la formación ético-cívica de los jóvenes a través de las TIC

Resumen. El presente artículo analiza la relevancia de la educación cívica en el contexto social actual, tomando como referencia el papel de las nuevas tecnologías y de la familia en la promoción y formación de actitudes cívicas en los jóvenes. Este trabajo se basa en un estudio bibliográfico, en el que se ha seguido un doble criterio: por un lado, se han seleccionado las publicaciones de los últimos cinco años que relacionan tecnologías, educación cívica y familia; y por otro lado, se ha tenido en cuenta la adecuación a los principales apartados de este artículo. La alfabetización digital de las familias y su participación en el contexto escolar se revela como una de las vías más adecuadas para transmitir a los hijos un aprendizaje en valores y un uso de las nuevas tecnologías que favorezca la participación cívica y una ciudadanía activa en la sociedad global.

Palabras clave: educación cívica; familia; TIC; ciudadanía; valores

Family involvement via ICT in young people's education in ethics and civics

Summary. This article analyzes the importance of civics education in the current social context, taking as its frame of reference the role of new technologies and the family in promoting civility among young people. This work is based on bibliographical study that followed two criteria: firstly, a selection was made of publications from the past five years that connect technology, civics education and the family, and secondly, a consideration was made as to the suitability for each of the sections in this article. Families' digital literacy and their participation in the school context is revealed to be one of the best ways to transmit values of civility to children and encourage in them a use of new technologies that favours participation in civic life and their active participation in global society.

Keywords: civics education; family; ICT; citizenship; values

Correspondencia:

Carmen Caro Samada

Gran Vía Rey Juan Carlos I, 41

26002 Logroño (La Rioja)

Teléfonos: 618567193 / 955225667

ccsamada@hotmail.com 


\section{Introducción}

La globalización en curso plantea a las sociedades actuales el reto de acometer empresas transnacionales, con repercusiones que también traspasan fronteras. La lógica de los mercados mundiales tiende a imponerse sobre las decisiones políticas de los gobiernos nacionales y, en consecuencia, afecta de modo desigual a diversos grupos sociales que configuran las mayorías parlamentarias. La consecuencia es una creciente desafección popular por los hasta ahora representantes de los partidos tradicionales (Levinson, 2012). Curiosamente, en un contexto en el que la tecnología permite mayor participación, la ciudadanía parece perder protagonismo en manos de técnicos que manejan datos y análisis de nivel macro.

La revitalización de la educación cívica tiene lugar en el contexto de crisis de la sociabilidad de nuestras sociedades, o de la democracia que requiere un fortalecimiento de la sociedad civil (Alvira, 2013). Ante las graves carencias que muchos ciudadanos presentan, con consecuencias negativas para la convivencia y el compromiso social, la educación cívica se muestra como una necesidad ineludible en el momento actual. Es en la década de los noventa cuando el debate sobre el tema comienza a tomar mayor protagonismo y a divulgarse, sobre todo en las discusiones teóricas entre liberales y comunitaristas en el ámbito anglosajón (MacIntyre, 2009; Rawls, 2000; Naval, 1995; Taylor, 1992; Waltzer, 1990; Mulhall \& Swift, 1992; Fowler, 1991).

La forja de una conciencia ciudadana activa requiere, en este contexto, una enseñanza adecuada a los nuevos tiempos, en la que se recupere la participación y el compromiso (Repáraz, Arbués, Naval \& Ugarte, 2015). A ello puede contribuir un uso responsable y apropiado de las nuevas tecnologías. Siendo un instrumento con enormes potencialidades, puede ser orientado tanto al uso individualizado y encorsetador (el solipsismo o la «endogamia digital» de la que habla Gozálvez, 2011, p. 134), como a un uso socializador, abierto y cooperativo que facilite el fortalecimiento de la conciencia ciudadana.

El objetivo de este artículo será reflexionar sobre el papel de las nuevas tecnologías en la educación cívica desde la educación formal, y, muy especialmente, en ámbitos informales, en este caso, la familia. Para ello se presenta una propuesta ético-cívica que favorezca la participación familia-escuela mediante el uso de las TIC.

\section{Metodología}

Al tratarse de un estudio mixto, teórico-bibliográfico, la elección de las fuentes de información ha sido el principal aspecto metodológico a considerar en el presente trabajo. Concretamente, hemos usado buscadores como GoogleScholar y bases de datos como Dialnet y Web of Science. En lo que respecta a los criterios para seleccionar publicaciones, se ha seguido un doble criterio: en primer lugar, la adecuación a los tres apartados centrales de este trabajo (ciudadanía y edu- cación, contexto tecnológico, familia y educación cívica), y, en segundo lugar, fecha de publicación tan reciente como sea posible. El objetivo de todo ello es fundamentar teóricamente la propuesta de la conexión familia-escuela-TIC para desarrollar la educación éticocívica de los estudiantes.

\section{Educación formal e informal: la importancia de la familia}

Pero ¿cómo se puede educar en ciudadanía? ¿Basta con una asignatura dentro del currículo? Siendo una actitud, y no tanto un conocimiento teórico, estudios de campo recientes hacen hincapié en la necesidad de un aprendizaje práctico, al considerar el concepto de ciudadanía, enmarcado en las virtudes sociales (Naval \& Arbués, 2015), como dinámico y relacional (Mata, Ballesteros \& Padilla, 2013). A través de una serie de entrevistas en profundidad, estos autores llegan a la conclusión de la pertinencia de modelos de prácticas ciudadanas (padres, profesores, compañeros, etc.), es decir, cauces de aprendizaje informal, para que la enseñanza formal de la ciudadanía tenga más probabilidades de éxito, al corresponderse con ejemplos de vida cercanos, pues la inmersión educativa es más adecuada que la transmisiva (Tonucci, 2009). En la misma línea se sitúan estudios sobre el aprendizaje del servicio (Stewart \& Bai, 2010), o propuestas como las comunidades prosociales (Tyler, 2004).

Las recomendaciones de la Unión Europea al respecto reúnen también los polos formal e informal de la enseñanza de la ciudadanía. Entre las finalidades que se pretenden lograr para construir una ciudadanía activa (Puig, Domene \& Morales, 2010) destacan: un modo pacífico de resolver conflictos, la defensa argumentada de la propia opinión, la escucha y comprensión de los demás, la aceptación de las diferencias, el análisis ético, la asunción de responsabilidades, la evitación de posturas agresivas, o el espíritu crítico. Finalidades que responden a la creciente apatía política, a la necesidad de fortalecer la confianza en las instituciones democráticas, de convivir en nuestras sociedades multiculturales, respetando a las minorías, promoviendo la tolerancia y la inclusión social.

En este sentido, la apuesta por la educación en competencias, en un contexto de «conocimiento líquido o esférico» (Valle, 2015), pone el acento en la responsabilidad del estudiante mediante un aprendizaje activo a través de proyectos colaborativos. Pero para que la transmisión de estos valores se internalice y se haga práctica, parece no solo oportuno, sino necesario, que la familia coopere con un profesorado adecuadamente formado y con un centro escolar constituido como amplia comunidad participativa (Fernández \& Álvarez, 2009).

En definitiva, llegar a ser ciudadano supone adquirir la competencia correspondiente, aprendida de modo práctico, a partir de modelos cercanos, que apliquen valores a la vida y enseñen a encarnar la enseñanza formal recibida en el sistema educativo. 


\section{Educar en ciudadanía en el sistema formal: escuela nueva, ética dialógica}

Entre las propuestas teóricas que acometen la formación moral y cívica como un fin del sistema educativo destacamos la Escuela Nueva y la ética dialógica. Dentro del movimiento de la Escuela Nueva, la solidaridad y la ciudadanía constituyen preocupaciones fundamentales (Fernández-Soria \& Mayordomo, 2014). El trabajo activo del niño, la cooperación y ayuda mutua, serían principios prioritarios frente a la obediencia o el interés individuales. Así, Dewey (1926) propone insistir en la construcción del saber, más que en su mero aprendizaje; y Kerschensteiner (1926) ve en el trabajo colectivo el fundamento para alcanzar el autogobierno, la responsabilidad y la forja de una comunidad moral. En definitiva, para la Escuela Nueva, la función social de la escuela sería clave, por encima de la instrucción intelectual a la que tradicionalmente se ha orientado el sistema educativo. Aun así, Piaget (1932) observa que la escuela debe fomentar dos clases de respeto: el unilateral, que procede de la moral heterónoma y que ha de primar en las edades tempranas, y el cooperativo, que procede de una moral autónoma, que debe desarrollarse en edades posteriores; $y$ ambos son fundamentales. En resumen, para la metodología de la Escuela Nueva, el autogobierno de obrar por sí mismo genera en el niño la necesidad de escoger entre el bien y el mal, adquiriendo así la responsabilidad moral. De igual modo, el cooperativismo hace que la verdadera educadora sea la vida colectiva (Profit, 1932). Sin embargo, el niño necesita unas referencias para escoger bien, primeramente familiares y posteriormente escolares. Por otra parte, el cooperativismo precisa de orientación al bien objetivo común, mostrado, a su vez, primero por la familia y luego por la escuela, pues también los miembros de una banda juvenil «cooperan» en el comportamiento desviado.

En cuanto a la ética dialógica, las sociedades plurales, democráticas e hipercomunicadas (Gozálvez \& Contreras, 2014) requieren la construcción de una ciudadanía mediática, que tenga por finalidad la reactivación de la sociedad civil; y para ello se necesita el aprendizaje de cauces de diálogo en el nuevo contexto tecnológico, que atiendan no solo a una alfabetización técnica, sino sobre todo moral, que dé sentido a un uso ético de la información, y de la participación autónoma en el diálogo cultural (Gutiérrez \& Tyner, 2012).

Para ello, la clave, una vez más, está en la formación del profesorado. Por eso, una formación ética y ciudadana debe impartirse con especial esmero en la universidad. Puesto que el pensamiento pedagógico acaba por impregnar la práctica, la formación del profesorado es una pieza fundamental en la transmisión efectiva de fines y hábitos ciudadanos. Un aprendizaje que fuera meramente profesional, técnico y científico, dejaría incompleta la formación del alumno. En este sentido, los profesores universitarios europeos reconocen que la formación universitaria es también ética y ciudadana, y procuran promoverla, frente a una actitud pre- dominantemente utilitarista y mercantilista en sus alumnos (Esteban, Mellén \& Buxarrais, 2014). El problema real se encuentra en la falta de evaluación de estas competencias. Reconocerlas como parte de la misión formativa universitaria en documentos oficiales, estatutos o normativas, sin cauces para su evaluación, las convierte en mera declaración de intenciones. En consecuencia, unos profesores poco evaluados en las competencias éticas y ciudadanas es posible que se ciñan a los textos oficiales disponibles sobre estas enseñanzas.

Pues bien, en cuanto al modo de presentar la educación en ciudadanía en los manuales más difundidos de la materia oficial, se advierte un enfoque liberal cosmopolita (González-García, 2012). Tanto en su vertiente cognitiva -de orientación ético-filosófica-, como en la afectiva y evaluativa, se incide en el ejercicio de derechos y obligaciones, con una metodología activa y participativa, si bien el debate y la reflexión crítica se desarrollan por medio de actividades personales más que grupales. Sobre ciertos valores básicos compartidos se procura la implicación de los alumnos en lo político como ámbito de decisión sobre el bien común, no circunscrito al mero ejercicio del derecho a votar.

Por tanto, desde el ámbito de la instrucción formal, la Escuela Nueva señala con acierto la relevancia de la formación en valores, si bien se centra en la autonomía cooperativa; por su parte, la ética dialógica añade la importancia del discurso y la participación con sentido. Se trata de una enseñanza que requiere mejorar las competencias prácticas del profesorado -no solo su evaluación teórica-, así como un enfoque de los manuales al uso que destaque la vertiente social y práctica de esta materia.

\section{Contexto tecnológico actual}

El contexto en el que se da tanto la formación del profesorado universitario como el despliegue de una asignatura específica sobre ciudadanía en la Educación Obligatoria, es el propio de una juventud socializada en el uso de las nuevas tecnologías (Bernete, 2009). Precisamente son las generaciones de nativos digitales las que tienden a manifestar mayor desconexión con la vida política, al menos en su versión institucional. Cabe considerar factores estructurales que inciden en el grado de participación política, como pueden ser, de un lado, un creciente neoliberalismo (Bauman, 2007; Couldry, 2010; Lewis, 2011), que sitúa en los mercados la clave técnica -más que política- de la toma de decisiones; y de otro, el incremento de movilizaciones a favor o en contra de la inmigración, de la pérdida de derechos sociales o, simplemente, de tipo antisistema. Todo ello manifiesta un cambio en el modelo de democracia, en la que los nuevos medios de información y de comunicación transforman el concepto de participación.

En efecto, las TIC, y su reciente evolución en la llamada Web 2.0, inciden cada vez más en los compor- 
tamientos y valores de la vida ordinaria. En general prevalece un uso comunicativo, lúdico y de consumo frente al directamente político; es más, los poderes tradicionales ya están presentes en el mundo on line, reproduciendo las relaciones de influencia habituales -como hemos podido comprobar en los últimos períodos electorales por la presencia de los líderes políticos en las redes-. Lo novedoso del nuevo entorno es la dificultad de poner límites a la intervención pública ciudadana; de ello se deduce una profunda alteración de «lo político». Pero lejos de reducirse al influjo directo del mundo digital, varios autores (Olsson \& Dahlgren, 2010; Livingston, 2009; Buckingham, 2007) advierten de una necesaria conexión entre el entorno on line y el off line entre los jóvenes digitales y los responsables de la toma de decisiones. Ciertamente el paradigma de lo público se altera en el momento en que Internet difunde y descentraliza la información, y rompe los límites de la comunicación. Aunque el activismo en la red va teniendo consecuencias en el comportamiento activo de las masas, los jóvenes internautas se manifiestan todavía «no muy solidarios y aún menos responsables» (Hernández-Prados, López \& Bautista, 2015, p. 183). Y es que, como otras tecnologías de gran potencial, las TIC ofrecen muchas posibilidades de intervenir en el poder como concepto relacional (Castells, 2009) facilitando su control; pero a la vez, pueden ser un instrumento de control férreo de la disidencia (Morozov, 2011).

En consecuencia, parece claro que las TIC ofrecen las condiciones para una participación de alcance nuevo, y que, a la vez, no bastan por sí mismas para generar un compromiso político efectivo. Se necesita además una «cultura cívica» (Dahlgren, 2011) cuyas bases se construyen en la escuela y sobre todo en la familia. En esta cultura cívica la red supone una fuente paralela de conocimiento, cuyo uso adecuado implica un reto pedagógico; puede también reflejar y fomentar valores democráticos como el respeto, la tolerancia o la transparencia; aun con sus peligros, Internet tiende a favorecer la confianza horizontal y la creación de vínculos nuevos. Pero todo ello precisa de la práctica, de competencias comunicativas que deben enseñarse aprovechando los nuevos espacios de intervención. De igual modo, junto con la disolución de la identidad en los mundos virtuales, las TIC facilitan la creación y el fortalecimiento de relaciones, la constitución de un «nosotros», una nueva identidad que actúa como unión entre la cultura cívica y la participación política. Se comprueba fácilmente que las cuestiones éticas están íntimamente involucradas en el uso apropiado y la implementación práctica de las tecnologías en el proceso de aprendizaje, hasta el punto de poder hablar de un «contexto ético continuo» (Olcott, Carrera, Gallardo \& González, 2015, pp. 62-63), que ponga la tecnología al servicio de las personas, valorando en cada situación práctica el comportamiento adecuado.

Dada la prioridad comunicativa que muestran los jóvenes en el uso de las TIC (Ballesteros \& Megías, 2015), especialmente manifiesto en las redes sociales virtuales, transformar y ampliar estos intercambios comunicativos en cauces de construcción de comunidad, supone «la necesidad de un marco educativo éticamente sintonizado» (Stornaiuolo, Dizio \& Hellmich, 2013, p. 87); con ello podríamos paliar la brecha entre las oportunidades de aprendizaje de las tecnologías y las limitadas prácticas con TIC en muchas familias. Dada la predisposición de los jóvenes a interaccionar de modo crítico y hospitalario, la institución educativa puede fomentar la producción colaborativa de un significado negociado y construido conjuntamente, algo fundamental en nuestro mundo global.

Un ámbito concreto en el que las TIC pueden contribuir a una mayor implicación de los jóvenes en la resolución de los problemas sociales es su implementación en el aprendizaje-servicio (Ugarte \& Naval, 2010; Santos-Rego, 2013; Middaugh \& Kahne, 2013). Las limitaciones de tiempo y espacio reglados por el sistema educativo pueden ser subsanadas mediante los nuevos medios tecnológicos y el apoyo familiar. A través de Internet se pueden construir experiencias de aprendizaje-servicio, simular la complejidad de los problemas sociopolíticos reales, promover comunidades virtuales en torno a cuestiones de justicia y equidad, en las que se oiga la voz de los jóvenes, o reflexionar sobre las consecuencias éticas que plantean tanto la red como la vida real. Mediante las redes sociales es más fácil superar la dicotomía activismo-pasividad y alcanzar diversos grados de compromiso (García-Galera, Del Hoyo \& Fernández-Muñoz, 2014), pues se pone de manifiesto que las proximidades geográfica, social y emocional se amplían como variables motivadoras del activismo on line. A falta de más estudios empíricos que evalúen la eficacia del uso de las TIC para el aprendizaje del compromiso ciudadano, e insistiendo en la necesidad de una mayor formación en su uso ético y social (García-Ruiz, Ramírez-García \& Rodríguez-Rosell, 2014), hay iniciativas on line (ColorOfChange, Adobe Youth Voices, TakingITGlobal, Fate of the World, Civilization, dosomething.org, Change.org, etc.) que ofrecen grandes oportunidades pedagógicas. La activación de estas oportunidades requerirá el concurso no solo de la escuela, sino también de la familia.

Asimismo habrá que evitar la paradoja del autismo tecnológico (Castells, 2009): la difuminación de límites objetivos compatible con la creación de límites subjetivos -contactar solo con los afines y, así, cerrarse en el propio grupo, ideología, gusto o prejuicio-. De ahí la importancia de la crítica constructiva y la cooperación interactiva. Se trata de trasladar al mundo virtual los modelos y las virtudes propias de la ciudadanía democrática. Y para ello, el aspecto ético es fundamental: profundizar en las dimensiones de la libertad, la igualdad, la solidaridad y el respeto, que promueve y es fruto del diálogo abierto (Gozálvez, 2011).

Precisamente la condición on line y virtual de las TIC, al tiempo que abre al conocimiento de otras realidades, añade un intermediario tecnológico, que puede distorsionar la realidad cotidiana o favorecer la evasión de ella. Destacamos en este campo diversas 
manifestaciones de adicción, como reacción a problemas sociales derivados del deterioro familiar (GomesFranco \& Sendín-Gutiérrez, 2014) o provocados por el bullying. Y a la vez, el mundo de las redes e Internet tiene un efecto disolvente de la responsabilidad moral (Hernández-Prados, Soriano, Fuentes \& Santos, 2015), como han puesto de manifiesto Arroyo y Gómez-Díaz (2015, p. 156) al hablar del peligro de una «incoherencia tecno-culturalmente inducida», en el sentido de disminuir la calidad de la respuesta moral ante un problema mediado virtualmente; de ahí la necesidad de educar en que los compromisos cara a cara, de la vida real, se mantengan igualmente al zambullirnos en la nube digital.

En definitiva, la educación en la ciudadanía activa encuentra en las nuevas tecnologías un apoyo instrumental de primer orden; sin embargo, la dimensión ética, que tanto la conciencia ciudadana como un uso adecuado de las TIC requieren, nos remite a cauces tanto formales como informales de aprendizaje, así como la necesidad de ejemplos de vida coherentes y cercanos. De ahí que familia y escuela se muestren como las instancias de socialización apropiadas para lograr esta finalidad.

\section{Familia y educación cívica}

Hablar de educación cívica supone, a su vez, abordar el papel de la institución familiar y su responsabilidad social en la educación social y cívica de sus miembros. Las medidas adoptadas por organismos como la ONU o el Consejo de Europa hacen hincapié en la cuestión de la familia, no solo desde un punto de vista privado sino también desde su repercusión pública. En este sentido, existe una continuidad entre la vida familiar y la vida social, recogida también desde los teóricos de la educación (Buxarrais \& Zeledón, 2006; Touriñán, 2010; Martínez \& Becedóniz, 2009). La crisis de sociabilidad que sufrimos en las sociedades contemporáneas ha hecho que volvamos la mirada a la familia. Esta recuperación ha estado ligada a la crítica feminista y muy especialmente a la comunitarista. Esta última vuelve a poner en primer plano las pequeñas comunidades, para subrayar la importancia del cuidado y atención a sus miembros (Naval, 2009). Por otra parte, desde 1994, que la UNESCO declaró «Año Internacional de la familia», las investigaciones en torno a la familia como institución social se han ido multiplicando (Alvira, 1998; Bas \& Pérez de Guzmán, 2010).

La familia se erige también como pilar para desarrollar dos dimensiones fundamentales en la educación cívica: la autonomía y la responsabilidad. Especialmente en el caso de la segunda, entendemos que la familia asume el proyecto de vida común, en el que todos los miembros se interrelacionan entre sí, y en el que cada uno de ellos es responsable del otro y con su entorno. La experiencia del amor incondicional puede ser escuela para el aprendizaje de la convivencia incondicionada.

Al hilo de esta cuestión, Bernal (2011) subraya el papel de la investigación sociológica en la revitalización de la familia como célula social básica. Destaca tres modalidades en las investigaciones actuales sobre responsabilidad social en la familia. Por un lado, la creación de contextos educativos familiares para facilitar el aprendizaje de la responsabilidad social. En segundo lugar, investigaciones sobre la prevención de conductas antisociales, asegurando las condiciones necesarias para formar sujetos responsables. En tercer y último lugar, los medios concretos para educar la responsabilidad social en la familia (como, por ejemplo, la implicación de los progenitores en actividades cívicas, conversaciones sobre temas sociales, apoyo a actividades de voluntariado o de aprendizaje-servicio, etc.).

Esta revitalización de la responsabilidad social de la familia, y su papel en la formación de actitudes cívicas, nos lleva a reivindicar su importancia en el contexto de la sociedad de la información. Los mass media no deben verse como enemigos, sino más bien como un recurso para fomentar una ciudadanía activa. Los espacios digitales pueden ser espacios privilegiados para la participación (Naval \& Arbués, 2015).

\section{TIC, educación en valores y familia}

Tal y como apunta Castells (2009), la Sociedad de la Información propicia que en los entornos culturales se revaloricen los procesos antes que los contenidos, que se comparta el valor de la comunicación antes que los valores compartidos; de ahí que sea necesario profundizar en el papel que pueden desempeñar las nuevas tecnologías para la transmisión y aprendizaje de los valores. Investigaciones como las de Pariente y Perochena (2013) ponen de relieve la eficacia del uso de las TIC para el aprendizaje de valores, especialmente el de justicia, centrado en los derechos y deberes de la ciudadanía, por considerarlo la base de los demás. Por su parte, el estudio de Hernández-Prados et al. (2015) llevado a cabo en alumnos de Educación Secundaria, señala que los adolescentes son capaces de identificar y de reconocer los valores que se transmiten en Internet: la creatividad, el diálogo, la colaboración y la participación son los más comunes. En cambio, el que menos reconocen es el de la honestidad. Esta misma investigación destaca también que las redes sociales se perciben como las mejores herramientas para la transmisión en valores. Para los adolescentes son más importantes los principios de cercanía en el mundo actual en el que viven, que los valores sociales universales (Royo, 2008). Por otro lado, se observa que los alumnos prefieren las actividades individualizadas en red, destinadas a satisfacer sus necesidades, antes que participar en comunidades colaborativas o instituciones destinadas a la ayuda a otros. Por último, manifiestan dificultad para cumplir con acuerdos establecidos con otros.

En los estudios mencionados la familia es percibida como el principal agente transmisor. Sin embargo, esto contrasta con investigaciones previas (HernándezPrados, López \& Sánchez, 2014; Bernete, 2009), que sugieren que la comunicación digital entre padres e hijos no se potencia lo suficiente, probablemente por 
la brecha digital y por la pérdida de privacidad que ello puede suponer.

Por su parte, Ruiz-Corbella y De Juanas (2013) señalan cómo las redes sociales han contribuido a una mayor participación social en Internet. En este sentido, la familia debe orientar en los valores y actitudes hacia una adecuada utilización de las redes sociales. Los jóvenes deben aprender a compartir espacios, en los que también se genera socialización. En consecuencia, la familia puede remodelar su relación con las TIC para aprovechar su propio rol socializador en valores, orientando las actividades tecnológicas de los hijos hacia un uso más colaborativo, en el que se afiancen los principios de la convivencia democrática activa.

Al hilo de esta idea conviene profundizar en las pautas de regulación que se llevan a cabo por parte de las familias, y las actitudes que estas desarrollan en relación con el uso de Internet de sus hijos. La investigación de Sureda, Comas y Morey (2010) constata que la regulación del uso de Internet por los menores se limita principalmente a restricciones de carácter temporal, de contenidos considerados inapropiados o como elemento de control según el comportamiento. Más de la mitad no estaría bajo ningún tipo de vigilancia. Ello indica que en la familia quedan relegados a un plano secundario los planteamientos y actitudes reguladoras, con un fin claramente cooperativo o participativo.

Por otro lado, la investigación de Ballesta y Cerezo (2011) subraya el papel de la cualificación de los padres sobre la valoración de las TIC, tanto en las escuelas como en el uso dentro del hogar. En este sentido la alfabetización audiovisual se manifiesta como una necesidad ineludible, constatada por los mismos progenitores. Así, el estudio de Guerra y Renés (2010) reveló que más de la mitad de las familias desconocía el significado de la alfabetización en medios o afirmaba no haber recibido formación sobre los mismos, y alrededor del 50\% manifestaba su interés en recibir formación periódicamente en este aspecto.

En definitiva, la mayor capacidad de interactuar que implican las TIC puede favorecer la adquisición de valores cívicos y cooperativos si los padres no se limitan a controlar el exceso de exposición a un mundo que desconocen, y rompiendo la brecha digital ejercen, también aquí, su papel orientador.

\section{Participación familia-escuela y TIC: una propuesta ético-cívica}

Tomando como referencia la concepción ecológica del desarrollo de Bronfenbrenner (1997), podríamos afirmar que en el nivel microsistémico -el del entorno inmediato del sujeto- muchos adultos no ofrecen un mapa de experiencia necesario para orientar en los códigos y en el uso responsable de las TIC. De ahí que el papel de los padres en este aspecto lo desempeñen, con frecuencia, otros agentes de socialización, como el grupo de amigos o incluso los intereses de las compañías tecnológicas.
El Consejo Escolar del Estado (2014) realizó recientemente una serie de recomendaciones sobre la influencia positiva de la participación de las familias en la educación escolar sobre el rendimiento académico de los alumnos. Las tomamos como referencia para arrojar luz sobre la participación familia-escuela y TIC, con el fin de orientarlas en sentido ético-cívico.

El Consejo Escolar resalta la importancia de realizar actividades en familia, en particular, dedicar todos los días un tiempo a conversar y compartir una comida principal. Actividades similares pueden ser realizadas en torno a las TIC: momentos de juego en familia, grupos familiares de WhatsApp, trabajos en Internet o la participación en alguna iniciativa solidaria de las ofrecidas en la red. Esta propuesta estaría en la línea de la de Hernández-Prados et al. (2014), quienes alertan de la falta de iniciativas para promover programas que favorezcan un uso responsable de las TIC. Plantean la necesidad de una agenda conjunta de ocio y tiempo libre que ofrezca un modelo positivo sobre uso de las redes: participación en foros especializados sobre cine o literatura, búsqueda de videotutoriales para manualidades, etc.

Otra de las indicaciones es la de situar las aspiraciones académicas de los propios hijos entre sus principales valores y supervisar las tareas escolares de los mismos. Esto se podría traducir en la supervisión de las tareas escolares que deban hacer on line, realizar tareas con los hijos en alguna de las web educativas interactivas (por ejemplo, http://www.aplicaciones.info/) o descubrir y suscitar aficiones, ampliando información disponible online, dándole un enfoque de aprendizaje.

Puede destacarse también la recomendación que hace hincapié en la necesidad de una formación de calidad en materia de participación escolar, dirigida a padres y profesores. Dicha formación podría orientarse a la mejora de la alfabetización digital. Por último, se trataría de aplicar estrategias que aumenten en los padres el sentimiento de pertenencia al centro escolar, factor clave en el rendimiento. En el tema que presentamos se aplicaría la formación en TIC a una mayor presencia on line de los padres en el centro escolar a partir de actividades, foros de discusión o de consulta, promoción de iniciativas familiares de curso, etc. No obstante, hacen falta más investigaciones en torno a la percepción de las familias y el nivel de implicación de las mismas a partir de la introducción de las nuevas tecnologías.

De hecho, en los últimos años se han promovido distintas iniciativas para integrar las tecnologías de la información y comunicación en los centros educativos. En el 2009 el Gobierno aprobó el proyecto Escuela 2.0 para que los alumnos hicieran uso de las nuevas tecnologías en el aula. Las TIC pueden ofrecer nuevas perspectivas para la participación de las familias y favorecer las relaciones entre familia, escuela y comunidad. Así, se señalan iniciativas como las escuelas de padres en la web, comunidades de aprendizaje, información a través de SMS, el correo electrónico, blogs o redes sociales (Aguilar \& Leiva, 2012). Las TIC se cons- 
tituyen en herramientas para promover una participación más activa y democrática en la escuela, no solo con el propio centro escolar, sino también con otros centros y familias, estableciendo redes de aprendizaje familiar. Una apuesta clave es la de fomentar así comunidades formativas de padres, en las que produzcan e intercambien materiales educativos on line (Zabalza, 2006). Se trata de un intercambio formativo, que ve a los miembros de la comunidad educativa no como meros consumidores sino también como productores. El compromiso con la comunidad educativa conlleva hacer frente a los problemas de la sociedad de una manera en la que los diferentes miembros se sienten copartícipes en la búsqueda de soluciones.

Otra propuesta interesante es la del Currículum Bimodal (Marqués \& Álvarez-Cánovas, 2014), que subraya la puesta en común que realizan los estudiantes en el aula de trabajo, con el fin de mejorarla entre todos y estimular las capacidades expresivas y argumentativas. En estos casos las herramientas tecnológicas, como la pizarra digital, resultan fundamentales. Al hilo de esta propuesta, Aguilar y Urbano (2014) plantean acciones en el aula acompañadas de actividades extraescolares en las que pueda colaborar la familia. Se trata de actividades lúdicas que el alumno puede realizar en la escuela y en casa. Algunas de ellas pueden ser visitas a museos, salidas o paseos, que favorecen la vida en familia.

\section{Discusión y conclusiones}

A la luz de las recomendaciones del Consejo de Estado sobre la influencia positiva de la participación de las familias en la educación escolar y sobre el rendimiento académico de los alumnos, cabe traducir las implicaciones de los estudios referidos en este artículo para el objetivo propuesto: la implicación de la familia en la formación ético-cívica de los jóvenes a través de las TIC.

Puesto que el aprendizaje cívico necesita de referentes cercanos, y dado que en el contexto diario de las nuevas generaciones está mediado por las tecnologías, parece poder concluirse la necesidad de que la familia tenga especial protagonismo. Aunque la brecha digital (Hernández-Prados et al., 2014) irá disminuyendo con el tiempo, se deberá seguir promoviendo la alfabetización mediática de los padres para que puedan cumplir con su papel como agentes de construcción de aprendizaje ciudadano.

Hay que tener en cuenta, como señala Bernete (2009), que la familia no puede pretender sustituir ni aun intervenir en algunas actividades que ya desarrollan los jóvenes en Internet, a las que ellos consideran ámbito privado de su personalidad, como aquellas que realizan con sus grupos de iguales. Pero sí cabe hacer presente en el mundo de las TIC una participación familiar en actividades solidarias, como se ha indicado. De igual modo podrían añadirse otros cauces para compartir actividades on line, como navegar y jugar en familia -con la consiguiente oportunidad de ofrecer modelos de comportamiento adecuados que prevengan o solucionen problemas como la adicción-, o proponer cauces on line paralelos a los habituales de los jóvenes (grupo familiar de WhatsApp, correo familiar, grupo familiar en redes sociales, blog familiar con fechas señaladas, etc.).

Otro ámbito en el que la familia puede, y debiera, involucrase más es el académico mediado por TIC. Propuestas como la alfabetización digital e intergeneracional de la familia en la escuela (Aguilar \& Urbano, 2014), a través de múltiples cauces (currículum bimodal, comunidades de aprendizaje, blogs compartidos, wikis, foros de discusión virtuales, etc.), pueden acercar la familia a la escuela, favoreciendo así la implicación de los padres y el mayor rendimiento de los hijos. En esas aspiraciones académicas de los alumnos, la supervisión familiar se sabe que ejerce un papel primordial para éxito escolar; las TIC pueden facilitar el seguimiento de las tareas, la coordinación con el colegio, la ampliación del apoyo mediante recursos didácticos interactivos, o suscitar y compartir aficiones a través de la web 2.0.

En todo este elenco de iniciativas, la presencia familiar constituye el necesario contrapunto de referencia para orientar el uso instrumental de las TIC hacia un uso con sentido, aprovechando las muchas posibilidades que ofrecen para la acción cooperativa, solidaria y de aprendizaje de valores. Empezando por aquellos comportamientos comprometidos en el modo de usar las TIC, se podrá avanzar en la promoción ulterior de un uso proactivo y cívico: que prime la solución de problemas del entorno, compartir inquietudes por el bien común, fomentar la participación en cuestiones sociales, la aportación crítica del propio punto de vista, el respeto y la tolerancia frente a otras opciones. En definitiva, un aprendizaje de los principios democráticos que genere conciencia social y responsabilidad personal, adaptadas al nivel de maduración de los hijos.

Es preciso superar el individualismo, el relativismo, la desesperanza, y favorecer el humanismo comunitario para ser capaces de compartir los bienes y recursos, incluidos los que ofrecen las nuevas tecnologías. Por ello, es necesaria una alfabetización acorde con los lenguajes, códigos y usos propios de la actual sociedad del conocimiento. Una sociedad en la que es preciso promover la solidaridad entre las generaciones; donde en las interacciones cotidianas los más jóvenes, hábiles en el manejo de las herramientas digitales, aporten su conocimiento digital a las generaciones adultas, de manera que favorezcan su inclusión en el uso diario de las nuevas tecnologías; y por su parte, las personas adultas aporten a los más jóvenes su experiencia y conocimiento en otros aspectos de la vida, también cruciales e importantes para ellos.

\section{Referencias}

Aguilar, M. C. \& Leiva, J. J. (2012). La participación de las familias en las escuelas TIC: análisis y reflexiones educativas. Pixel-Bit. Revista de Medios y Educación, 40, 7-19. 
Aguilar, M. C. \& Urbano, A. (2014). La necesidad de alfabetización digital e inter-generacional en la familia y la Escuela. Revista DIM, 28, 1-16.

Alvira, R. (1998). El lugar al que se vuelve: reflexiones sobre la familia. Pamplona: EUNSA.

Alvira, R. (2013). La sociedad civil como base de la educación y la educación como base de la sociedad civil. Revista Española de Pedagogía, 71(254), 147-154.

Arroyo, I. \& Gómez-Díaz, R. (2015). Efectos no deseados por la comunicación digital en la respuesta moral. Comunicar, 22(44), 149-158. DOI: 10.3916/C44-2015-16

Ballesta, J. \& Cerezo, M. C. (2011). Familia y escuela ante la incorporación de las tecnologías de la información y la comunicación. Educación XX1, 14(2), 133-156. DOI: 10.5944/educxx1.14.2.248

Ballesteros, J. C. \& Megías, I. (2015). Jóvenes en la red. Un selfie. Madrid: Centro Reina Sofía sobre Adolescencia y Juventud.

Bas, E. \& Pérez de Guzmán, M. V. (2010). Desafíos de la familia actual ante la escuela y las tecnologías de información y comunicación. Educatio Siglo XXI, 28(1), 41-68.

Bauman, Z. (2007). Liquid Times: Living in an Age of Uncertainty. Cambridge: Polity Press.

Bernal, A. (2011). Responsabilidad social y educación en la familia: nuevos enfoques. XII Congreso Internacional de Teoría de la Educación, Universidad de Barcelona.

Bernete, F. (2009). Usos de las TIC, relaciones sociales y cambios en la socialización de las y los jóvenes. Revista de estudios de juventud, 88, 97-114. Recuperado el 25 de octubre de 2015 de http://goo.gl/yJHhcG

Bronfenbrenner, U. (1997). El sentido ecológico del desarrollo humano. México: Paidós.

Buckingham, D. (Ed.) (2007). Youth, Identity and the Digital Media. Cambridge. Massachusetts: MIT Press.

Buxarrais, M. R. \& Zeledón, M. P. (Coords.) (2006). Las familias y la educación en valores democráticos. Retos y perspectivas actuales. Barcelona: Claret.

Castells, M. (2009). Comunicación y poder. Madrid: Alianza Editorial.

Consejo Escolar del Estado (2014). La participación de las familias en la educación escolar. Madrid: Ministerio de Educación, Cultura y Deporte. Recuperado el 15 de octubre de 2015 de http://goo.gl/iYqY5c

Couldry, N. (2010). Why Voice Matters: Culture and Politics After Neoliberalism. Londres: Sage.

Dahlgren, P. (2011). Jóvenes y participación política: los medios en la Red y la cultura cívica. Telos: Cuadernos de comunicación e innovación, 89, 12-22.

Dewey, J. (1926). Ensayos de educación. Madrid: La Lectura.

Esteban, F., Mellén, T. \& Buxarrais, M. R. (2014). Concepciones del profesorado universitario sobre la formación ética y ciudadana en el Espacio Europeo de Educación Superior: un estudio de caso. RUSC. Universities and Knowledge Society Journal, 11(3), 22-32. DOI: 10.7238/rusc.v11i3.1778

Fernández-Soria, J. M. \& Mayordomo, A. (2014). Educación moral y educación cívica. Aportaciones de la Escuela Nueva. Estudios sobre educación, 26, $197-$ 217.

Fernández, M. D. \& Álvarez, Q. (2009). Un estudio de caso sobre un proyecto de innovación con TIC en un centro educativo de Galicia: ¿acción o reflexión? Bordón, 61(1), 95-108.

Fowler, R. B. (1991). The Dance with Community: The Contemporary Debate in American Political Thought. Kansas City: University of Kansas City.

García-Galera, M. C., Del Hoyo, M. \& Fernández-Muñoz, C. (2014). Jóvenes comprometidos en la Red: el papel de las redes sociales en la participación social activa. Comunicar, 22(43), 35-43. DOI: 10.3916/C432014-03

García-Ruiz, R., Ramírez-García, A. \& Rodríguez-Rosell, M. M. (2014). Educación en alfabetización mediática para una nueva ciudadanía prosumidora. Comunicar, 43(22), 15-23. DOI: 10.3916/C43-2014-01

Gomes-Franco, F. \& Sendín-Gutiérrez, J. C. (2014). Internet como refugio y escudo social: Usos problemáticos de la Red por jóvenes españoles. Comunicar, 22(43), 45-53. DOI: 10.3916/C43-2014-04

González-García, E. (2012). Educación de la cultura política en los manuales escolares de Educación para la Ciudadanía y los Derechos Humanos. Profesorado, 16(2), 501-516.

Gozálvez, V. (2011). Educación para la ciudadanía democrática en la cultura digital. Comunicar, 36(18), 131-138. DOI: 10.3916/C36-2011-03-04

Gozálvez, V. \& Contreras, P. (2014). Empoderar a la ciudadanía mediática desde la educomunicación. Comunicar, 21(42), 129-136. DOI: 10.3916/C42-2014-12

Guerra, S. \& Renés, P. (2010). La formación en medios y pantallas de las familias. Pixel-Bit. Revista de Medios y Educación, 36, 193-202.

Gutiérrez, A. \& Tyner, K. (2012). Educación para los medios, alfabetización mediática y competencia digital. Comunicar, 38, 31-39. DOI: 10.3916/C38-201202-03

Hernández-Prados, M. A., López, P. \& Bautista, V. (2015). La percepción del alumnado de educación secundaria sobre la transmisión de valores a través de las TIC. Teoría de la Educación. Revista Interuniversitaria, 27(1), 169-185. DOI: 10.14201/teoredu2015271169185

Hernández-Prados, M. A., López, P. \& Sánchez, S. (2014). La comunicación en la familia a través de las TIC: percepción de los adolescentes. Pulso: Revista de Educación, 37, 35-58.

Hernández-Prados, M. A., Soriano, E., Fuentes, J. L. \& Santos, M. (2015). Retos de la educación de nuestro siglo: Interculturalidad, responsabilidad tecnológica, y educación para la vida. En J. L. González Geraldo (Coord.), Educación, desarrollo y cohesión social (pp. 317-346). Cuenca: Universidad de CastillaLa Mancha.

Kerschensteiner, G. (1926). El autogobierno de los alumnos. Revista de Pedagogía, 49, 18-24.

Levinson, M. (2012). No citizen left behind. Cambridge, MA: Harvard University Press. 
Lewis, J. (2011). Crisis in the Global Mediasphere: Desire, Displeasure and Cultural Transformation. Basingstoke: Palgrave.

Livingston, S. (2009). Children and the Internet. Cambridge: Polity Press.

MacIntyre, A. (2009). Tras la virtud. Barcelona: Crítica.

Marqués, P. \& Álvarez-Cánovas, I. (2014). El currículo bimodal como marco metodológico y para la evaluación. Principios básicos y mejoras obtenidas en aprendizajes y rendimiento de los estudiantes. Educar, 50(1), 149-166.

Martínez, R. A. \& Becedóniz, C. M. (2009). Orientación Educativa para la Vida Familiar como Medida de Apoyo para el Desempeño de la Parentalidad Positiva. Intervención Psicosocial, 18, 97-112.

Mata, P., Ballesteros, B. \& Padilla, M. T. (2013). Ciudadanía participativa y transformadora: análisis de discursos y propuestas de aprendizaje. Teoría de la Educación. Revista Interuniversitaria, 25(2), 49-68.

Middaugh, E. \& Kahne, J. (2013). Nuevos medios como herramienta para el aprendizaje cívico. Comunicar, 40(20), 99-108. DOI: 10.3916/C40-2013-02-10

Morozov, E. (2011). The Net Delusion: How Not to Liberate the World. Londres: Allen Lane.

Mulhall, S. \& Swift, A. (1992). Liberals and Communitarians. Oxford: Blackwell.

Naval, C. (1995). La controversia liberal-comunitarista en educación. Enrahonar. Quaderns de Filosofía, 24, 81-97.

Naval, C. (2009). Ámbito familiar: confianza y respeto. En A. Bernal (Ed.), La familia como ámbito educativo (pp. 145-162). Madrid: Rialp.

Naval, C. \& Arbués, E. (2015). Del uso de Internet en la promoción de virtudes sociales. Un caso concreto: Parlamento Cívico. Teoría de la Educación. Revista Interuniversitaria, 27(1), 33-52. DOI: 10.14201/teoredu20152713352

Olcott Jr., D., Carrera, X., Gallardo, E. E. \& González, J. (2015). Ética y Educación en la era digital: perspectivas globales y estrategias para la transformación local en Cataluña. RUSC. Universities and Knowledge Society Journal, 12(2), 59-72. DOI: 10.7238/rusc. v12i2.2455

Olsson, T. \& Dahlgren, P. (Eds.) (2010). Young People, ICTs and Democracy. Gotemburgo: Nordicom.

Pariente, J. L. \& Perochena, P. (2013). Didáctica de la educación en valores en la ESO. Una propuesta utilizando las tecnologías para el aprendizaje y el conocimiento. Pixel-Bit, 42, 195-208.

Piaget, J. (1932). Los procedimientos de la educación moral. En P. Petersen \& J. Piaget, La nueva educación moral (pp. 39-93). Madrid: Publicaciones de la Revista de Pedagogía.

Profit, B. (1932). La coopération scolaire française. Pour l'Ère Nouvelle. Revue Internationale d'Éducation Nouvelle, 79, 183-184.

Puig, M., Domene, S. \& Morales, A. (2010). Educación para la ciudadanía: referentes europeos. Teoría de la Educación. Revista Interuniversitaria, 22(2), 85-110.
Rawls, J. (2000). Teoría de la Justicia. México D.F.: Fondo de Cultura Económica.

Repáraz, C., Arbués, E., Naval, C. \& Ugarte, C. (2015). El Índice Cívico de los universitarios. Sus conocimientos, actitudes y habilidades de participación social. Revista Española de Pedagogía, 73(260), 23-52.

Royo, J. (2008). Los rebeldes del bienestar: Claves para la comunicación con los nuevos adolescentes. Barcelona: Alba Editorial.

Ruiz-Corbella, M. \& De Juanas, A. (2013). Redes sociales, identidad y adolescencia: nuevos retos educativos para la familia. Estudios sobre Educación, 25, 95-113.

Santos-Rego, M. Á. (2013). ¿Para cuándo las universidades en la agenda de una democracia fuerte? Educación, aprendizaje y compromiso cívico en Norteamérica. Revista de Educación, 361, 565-590. DOI: 10.4438/1988-592X-RE-2013-361-233

Stewart, T. \& Bai, H. (2010). Community Service SelfEfficacy and Summer Service-Learning: Comparative Analyses Among Academically Talented Youth. Gifted Education International, 27(2), 149-160. DOI: 10.1177/026142941002700205

Stornaiuolo, A., Dizio, J. \& Hellmich, E. (2013). Desarrollando la comunidad: jóvenes, redes sociales y escuelas. Comunicar, 20(40), 79-88. DOI: 10.3916/ C40-2013-02-08

Sureda, J., Comas, R. \& Morey, M. (2010). Menores y acceso a Internet en el hogar: las normas familiares. Comunicar, 27, 135-143. DOI: 10.3916/C34-201003-13

Taylor, C. (1992). Multiculturalism and the Politics of Recognition. Nueva Jersey: Princeton University Press. Tonucci, F. (2009). ¿Se puede enseñar la participación? ¿Se puede enseñar la democracia? Investigación en la Escuela, 68, 11-24.

Touriñán, J. M. (2010). Familia, escuela y sociedad civil. Agentes de educación intercultural. Revista de investigación en educación, 7, 7-36.

Tyler, F. B. (2004). The role of prosocial communities in youth development. Psykhe, 13(2), 3-15.

Ugarte, C. \& Naval, C. (2010). El service-learning, medio adecuado para promover la cooperación social. El caso concreto del programa Create your own charity. En C. Naval, S. Lara, C. Ugarte \& C. Sádaba (eds.), Educar para la comunicación y la cooperación social (pp. 263-281). Pamplona: CoAN.

Valle, J. M. (2015). Aprendizaje para la sociedad de hoy. Enseñar por competencias. Ponencia presentada en el XII Congreso Internacional de Educación y Aprendizaje. Madrid: CEU.

Waltzer, M. (1990). The Communitarian Critique of Liberalism. Political Theory, 18(1), 6-23.

Zabalza, M. (2006). Buscando una nueva hoja de ruta en la formación. Revista de Educación, 340, 51-58.

\section{La implicació de la família en la formació ètica i cívica dels joves a través de les TIC}

Resum. El present article analitza la rellevància de l'educació cívica en el context social actual, prenent com a referència el 
paper de les noves tecnologies $i$ de la família en la promoció $i$ formació d'actituds cíviques en els joves. Aquest treball es basa en un estudi bibliogràfic, en el qual s'ha seguit un doble criteri: d'una banda, s'han seleccionat les publicacions dels últims cinc anys que relacionen tecnologies, educació cívica i família; $i$ d'altra banda, s'ha tingut en compte l'adequació als principals apartats d'aquest article. L'alfabetització digital de les famílies i la seva participació en el context escolar es revela com una de les vies més adequades per transmetre als fills un aprenentatge en valors $i$ un ús de les noves tecnologies que afavoreixi la participació cívica i una ciutadania activa en la societat global.

Paraules clau: educació cívica; família; TIC; ciutadania; valors 